Gefässchirurgie 2015 $20: 299-300$

DOI 10.1007/s00772-015-0030-9

(C) Springer-Verlag Berlin Heidelberg 2015

\title{
C. Baethge
}

Leiter der Medizinisch-Wissenschaftlichen Redaktion, Deutsches Ärzteblatt

und Deutsches Ärzteblatt International, Köln, Deutschland

\section{Die Interessen von Lesern und Autoren wiedervereinigen}

wa randomisierte Studien oder systematische Übersichtsarbeiten, selten sind $[1,3]$.

So haben Galandi et al. [3] festgestellt, dass seit den achtziger Jahren kaum noch randomisierte Studien auf Deutsch publiziert wurden. Nach meiner Wahrnehmung gilt dies aber auch für hochwertige epidemiologische Originalarbeiten oder systematische Übersichtsarbeiten. Das hat dazu geführt, dass viele deutschsprachige Periodika die Publikationssprache gewechselt haben und nunmehr auf Englisch erscheinen. Ein Beispiel aus meinem eigenen Fach, der Psychiatrie, soll die Entwicklung verdeutlichen.

\section{Englisch- versus deutschsprachige Fachzeitschriften}

In den achtziger Jahren des Zwanzigsten Jahrhunderts hat sich das 1868 gegründete und damals führende Archiv für Psychiatrie und Nervenkrankheiten in eine englischsprachige Zeitschrift verwandelt. Heute heißt sie European Archives of Psychiatry and Clinical Neuroscience, und sie liegt mit einem Impact-Faktor von rund 3,4 auf Platz 40 in der Rangliste aller psychiatrischen Journale mit Impact-Faktor, dem durch die auf ein Journal entfallenden Zitate errechneten Bewertungsmaß für Fachzeitschriften.

Die European Archives sind in gewisser Weise eine deutsche Zeitschrift geblieben. Sie wird von einem deutschen Verlag publiziert (Springer) und ist personell eng mit der deutschsprachigen Universitätspsychiatrie verflochten: So hat sie einen Herausgeber aus München und fast die Hälfte der Mitglieder des Wissenschaftlichen Beirats kommen aus Deutschland. In der April-Ausgabe stammten sieben von elf Beiträgen federführend aus deut- schen oder schweizer Institutionen. Die Zeitschrift hat es international zu Ansehen gebracht. Aber nach meiner Wahrnehmung lesen die hiesigen Psychiater selbst universitäre - sie nicht häufiger als andere internationale Zeitschriften, nämlich abgesehen von ihren Spezialthemen relativ selten. Mit anderen Worten: Ihr Einfluss in Deutschland ist nicht groß.

Demgegenüber hat der auf Deutsch erscheinende Nervenarzt als größte psychiatrische Fachzeitschrift in Deutschland nach Verlagsangaben eine verkaufte Auflage von über 14.000. Das Journal erfreut sich also erheblicher Beliebtheit und - so nehme ich es wahr - wird verbreitet gelesen. Der Impact-Faktor von 0,7 zeigt aber seine Randstellung in der globalen wissenschaftlichen Psychiatrie (Platz 106). Das Beispiel zeigt, dass man mit einer englischsprachigen Zeitschrift eine gewisse wissenschaftliche Reputation erlangen kann, dabei aber nicht die breite Masse der Leser erreicht. Wenn man aber umgekehrt ein deutschsprachiges Periodikum herausgibt und viele Leser unter den einheimischen Ärzten findet, muss man auf den wissenschaftlichen „Impact" verzichten.

Dieser Befund lässt sich auf andere nicht englischsprachige Publikationen übertragen: In einer eigenen Studie hat sich gezeigt, wie wenig die nichtenglischsprachige wissenschaftliche Publizistik international wahrgenommen wird [1]: Nur die Hälfte aller im Jahr 2009 in zehn nichtenglischsprachigen psychiatrischen Periodika erschienenen knapp 200 Originalarbeiten wurde in den darauf folgenden zwei Jahren überhaupt zitiert, insgesamt $246 \mathrm{Mal}$. Von diesen Zitaten erschien nur rund ein Drittel aller Zitate in englischsprachigen Zeitschriften. 


\section{Die Zuneigung zur Muttersprache}

Warum aber haben bei uns deutschsprachige Zeitschriften so viel mehr Leser? Aus einer Umfrage unter Allgemeinmedizinern wissen wir, dass sieben von zehn es für wichtig oder sehr wichtig halten, dass Fortbildungen auf Deutsch erscheinen [5]. Solche Einstellungen sind wirkungsmächtig: Das zeigt sich beispielsweise an der mit rund 160 niedrigen Zahl individueller Abonnenten, die das British Medical Journal, zumindest vor einigen Jahren in Deutschland zählte (Geetha Balasubramaniam, British Medical Journal, persönliche Mitteilung, Dezember 2007) - und dies, obwohl es sich um eine für Allgemeinärzte maßgeschneiderte Zeitschrift von hohem internationalen Niveau handelt.

Trotz der Fremdsprachenkenntnisse, die man voraussetzen kann, halten viele Ärzte am Deutschen fest, weil sie darin sicherer sind. In unserer Muttersprache lesen und schreiben wir in der Regel am besten. So haben beispielsweise Gulbrandsen et al. [4] in einer randomisierten Studie gezeigt, dass der Lernerfolg selbst bei den für ihre Englischkenntnisse gepriesenen Skandinaviern nennenswert und statistisch signifikant besser war, wenn sie einen Artikel in ihrer Muttersprache statt auf Englisch gelesen hatten.

\section{Die Folgen: „Wir leben von der Hand in den Mund"}

Es zeigt sich also eine Dissoziation zwischen der wissenschaftlich hochwertigen Publizistik, die auf Englisch stattfindet, und den Lektürebedürfnissen der überwiegend klinisch tätigen Ärzteschaft. Wäre dies nur eine wertneutrale Entwicklung in einer sich differenzierenden Publikationslandschaft, könnte man es bei der Feststellung dieser Dissoziation belassen. Da aber mit dieser Auseinanderentwicklung auch die Qualität der Fachzeitschriften verbunden ist, ergibt sich aus meiner Sicht Handlungsbedarf. Es ist ja nicht nur so, dass die wichtigsten Originalarbeiten auf Englisch erscheinen. Nach meiner Erfahrung fällt es den Schriftleitern vieler deutschsprachiger Zeitschriften oft auch schwer, Autoren für gute Über- sichtsarbeiten zu gewinnen. Vor kurzem fasste es ein mir bekannter Redakteur so zusammen: „Wir leben von der Hand in den Mund“.

\section{Eine Lösung: Bilinguales Publizieren}

Keiner könnte, und vermutlich wollte auch niemand die Entwicklung zum Englischen als Lingua franca der Wissenschaft rückgängig machen. Es ist ein zivilisatorischer Fortschritt, dass Ärzte und Wissenschaftler weltweit eine gemeinsame Sprache nutzen. Wenn aber englischsprachige Zeitschriften in Deutschland wenig gelesen werden und wenn deutschsprachige Zeitschriften wissenschaftlich marginalisiert sind, weil sie für Autoren unattraktiv geworden sind, bietet sich aus meiner Sicht vor allem ein Ausweg an: die komplett zweisprachige Publikation einer medizinischen Fachzeitschrift.

Es gibt mittlerweile einige Zeitschriften, die auf Englisch und in der Muttersprache publizieren. In Deutschland trifft dies etwa auf das Journal der Deutschen Dermatologischen Gesellschaft (JDDG) zu und auf das Deutsche Ärzteblatt mit seinem Online-Ableger Deutsches Ärzteblatt International. Andere Beispiele wären das Jornal de Pediatria aus Brasilien oder die Archivos de Bronconeumologia aus der spanischsprachigen Welt. Der Schriftleiter des Jornal de Pediatria berichtete, dass sich der Schritt in die Zweisprachigkeit für sie gelohnt habe. So sei die Zeitschrift in Medline aufgenommen worden, eine Datenbank, die wegen der damit verbundenen internationalen Sichtbarkeit für Autoren große Bedeutung hat, und hätte mehr und bessere Manuskripte erhalten [2].

Für das Deutsche Ärzteblatt ist Ähnliches festzustellen. Nach zuvor mehreren vergeblichen Anläufen erfolgte erst mit der zweisprachigen Ausgabe eine Aufnahme in Medline. Dies trifft auch auf den Journal Citation Report zu, wodurch das Ärzteblatt auch einen Impact-Faktor erhielt. All das hat uns für Autoren attraktiver gemacht, sodass die Zahl der eingehenden Manuskripte deutlich gestiegen ist und wir hoffen, unseren Lesern künftig noch bessere Artikel anbieten zu können.
Zweisprachiges Erscheinen ist mit Aufwand verbunden: Übersetzungen kosten Geld und die redaktionelle Qualitätskontrolle ist ressourcen-, arbeitsund zeitintensiv. Ich bin daher dankbar, dass sich die Herausgeber des Deutschen Ärzteblattes, die Bundesärztekammer (BÄK) und die Kassenärztliche Bundesvereinigung (KBV) entschlossen haben, die zweisprachige Ausgabe des wissenschaftlichen Teils des Deutschen Ärzteblattes zu fördern. Dies gilt auch für das Engagement des Deutschen Ärzte-Verlages. Insgesamt kann man aber sagen, dass sich dieses Engagement für alle Beteiligten gelohnt hat - vor allem für die Leser und für die Autoren.

\section{Korrespondenzadresse

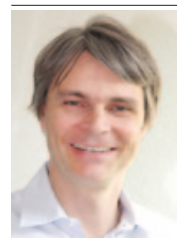 \\ Prof. Dr. C. Baethge Leiter der Medizinisch- Wissenschaftlichen Redaktion Deutsches Ärzteblatt und Deutsches Ärzteblatt International Ottostraße 12, 50589 Köln baethge@aerzteblatt.de}

\section{Einhaltung ethischer Richtlinien}

Interessenkonflikt. C. Baethge ist Leiter der Medizinischen Wissenschaftlichen Redaktion von Deutsches Ärzteblatt und Deutsches Ärzteblatt International.

\section{Literatur}

1. Baethge C (2013) The production and recognition of psychiatric original articles published in languages other than English. BMC Psychiatry 13:102. doi:10.1186/1471-244X-13-102

2. Blank D, Buchweitz C, Procianoy R (2005) Impact of SciELO and MEDLINE indexing on the submission of articles to a "non-English" journal. Abstracts of the 5th International Congress on Peer Review and Biomedical Publication. Chicago, USA

3. Galandi D, Schqarzer G, Antes G (2006) The demise of the randomised controlled trial: bibliometric study of German-language health care literature, 1948-2004. BMC Med Res Methodol 6:30

4. Gulbrandsen P, Schroeder TV, Milerad J, Nylenna M (2002) Paper or screen, mother tongue or English: which is better? JAMA 287:2851-2853

5. Ruf D, Berner MM, Kriston L, Maier I, Härter M (2008) Hausärzte online: Gute Voraussetzungen, aber geringe Nutzung des Internets zur Fortbildung. Z Evid Fortbild Qual Gesundh Wesen 102:291-297 